\title{
How much wrapping do babies need at night?
}

\author{
Ruth E Wigfield, Peter J Fleming, Yehu E Z Azaz, Thelma E Howell, Debbie E Jacobs, \\ Pam S Nadin, Rosie McCabe, Alison J Stewart
}

\begin{abstract}
In a longitudinal, population based study, overnight temperature recordings were made in the bedrooms of 152 babies aged 3-18 weeks and the insulation provided by their bedclothing was assessed. Outdoor temperatures for the study nights were also available.

Parents applied more insulation on colder nights with lower bedroom temperatures than on warmer nights (mean 8.5 tog at $15^{\circ} \mathrm{C}$ minimum bedroom temperature falling to 4.0 tog at $25^{\circ} \mathrm{C}$ ). For a particular temperature they also applied 2 tog more insulation in winter than in summer.

The amounts of bedclothing used in the home were compared with insulation levels predicted to achieve thermoneutrality over a similar range of environmental temperature from heat balance studies in young infants. They corresponded closely.

The average amount of bedclothing chosen for babies in Avon allows them to remain in thermoneutral conditions throughout the night. These values are proposed as broad guidelines for the thermal care of young babies at night. (Arch Dis Child 1993; 69: 181-186)
\end{abstract}

Thermal stress may be a contributory factor in some sudden infant deaths. The first indication of this came from Stanton's recognition of various risk factors for overheating in the histories of many cot death victims. ${ }^{1}$ These original anecdotal observations were later confirmed in two separate casecontrol studies from Avon in the $\mathrm{UK}^{2}$ and from Tasmania. ${ }^{3}$ In the Avon study, the infants who died were more heavily wrapped and more likely to have had the heating left on overnight than closely matched living controls. The Tasmanian findings were similar, in that the infants who died were again more heavily wrapped and they were found in warmer rooms than matched controls.

The data from these studies raise the question of what are appropriate thermal conditions for young babies to sleep under. What is the optimal temperature range for the bedroom and how much clothing and bedding should be used? In an attempt to address this question we have compared the results of two studies carried out independently in Avon. The first was a descriptive study of what was actually happening in our community. It was undertaken by measuring bedroom temperatures in babies' homes and by discovering the type and amount of clothing and bedding that babies were sleeping under, throughout the year and longitudinally over the first few months of life. In contrast the second study was carried out in a laboratory environment using a series of heat balance measurements on a smaller number of babies to determine how much insulation was required to achieve thermoneutrality over a range of environmental temperatures comparable to those in the home.

\section{Methods}

COMMUNITY METHODS

As part of a more extensive study of infant care practices and mortality, a population based group of normal babies was identified by the systematic selection of every 125 th baby born to Avon residents over the 18 month period between January 1990 and June 1991. The babies' parents were contacted via their health visitors and they were subsequently visited at home on up to three occasions during the first six months of life. If the 125 th baby were unavailable for study, the 124th or 126th baby from the birth records was substituted to ensure that the seasonal pattern of births in the study sample was representative of the whole birth cohort.

At each visit a questionnaire was completed including detailed information about the clothing and bedding used for the baby at night and the heating arrangements in the home. Overnight temperature recordings were made from four sites around the baby's usual sleeping place: two recorded the surface temperature of the two walls closest to the baby and the other two recorded air temperature over the sleeping place. The measurements were automatically taken at one minute intervals and stored in a portable, digital datalogger (Squirrel model 1202, Grant Instruments (Cambridge) Limited). The average temperature of all four probes during the coolest and the warmest five minute periods of the night were taken as the minimum and maximum bedroom temperatures respectively. The daily outdoor minimum temperatures measured at three sites 
within the county of Avon were obtained from the Meteorological Office (Bracknell, UK).

During the overnight study the parents completed a diary sheet recording the amount and type of clothing and bedding used and the baby's location and activity. From this information the effective thermal resistance of all the coverings was assessed in togs using the method reported by Fleming et $a l^{2}$ which takes account of both the intrinsic thermal resistance of the fabric and the relative surface area covered by each item. It is difficult to ascribe an accurate tog value to a nappy as the thermal resistance of the fabric is variable depending on whether it is wet or dry. In both situations disposable nappies provide the same thermal insulation as towelling ones (approximately $0 \cdot 1$ tog wet and 0.5-0.6 tog dry). As every baby wore a nappy and the value of the insulation it provided, wet or dry, was small compared with the overall value we have excluded nappies from our estimations.

\section{LABORATORY METHODS}

The laboratory heat balance studies were performed in a modified barometric plethysmograph that allows accurate measurement of oxygen consumption and respiration to be made in relatively unrestrained infants. The equipment and method have been published in detail elsewhere. ${ }^{4}$ The metabolic rate was calculated from the measurements of oxygen consumption and the evaporative water losses from both the respiratory tract and the baby's skin were determined.

Twenty two healthy, full term babies were studied on several occasions from 2 days to 3 months of age. For the present investigation, the results obtained from recordings made within the first week of life and at 3 months of age have been used.

\section{HEAT BALANCE EQUATIONS}

The range of environmental temperature over which a minimal metabolic rate is maintained, that is the thermoneutral range, is dependent on the amount of insulation covering the body. The previously accepted concept of the thermoneutral range also held that the evaporative water losses were minimal throughout the range. This has recently been shown to be incorrect, ${ }^{4}$ and in fact evaporative water loss starts to rise at an environmental temperature below the lower critical temperature and continues rising throughout the thermoneutral range. Therefore to keep the total heat loss (equal to the metabolic rate) constant, the non-evaporative components of heat loss must decrease with increasing environmental temperature across the thermoneutral range. In the previously published studies we were unable to define reliably the upper end of the thermoneutral range because of wide interindividual variations. ${ }^{4}$ However, within the conditions of that study (in which infants were covered with a total of 2.5 tog of bedding and clothing) the highest environmental temperature that we could clearly demonstrate as being within the thermoneutral range was $27^{\circ} \mathrm{C}$. This has therefore been taken as the upper temperature for the purposes of the present study. It does not represent the upper critical temperature but one within and close to the upper end of the thermoneutral range. Values of non-evaporative heat loss measured both at the lower critical temperature and close to the upper limit of the thermoneutral range were used in the heat balance equation detailed in the appendix to determine the amounts of insulation required to allow babies to remain within the thermoneutral range over a wide range of environmental temperatures. The validity of this technique was also checked by solving the equation individually for each infant, using the actual air temperatures measured in their study, and comparing the level of insulation calculated to be present with the known amount of bedding and clothing used in the study.

\section{Results}

COMMUNITY RESULTS

A total of 152 babies were recruited into the community study of whom 100 were of the original selection and 52 were substitutes. The sex ratio of the study subjects (79 boys and 73 girls) was similar to that of all Avon births but the social class distribution was skewed slightly upwards by the 52 substitutes compared with the babies they were replacing.

In this paper, for comparison with the laboratory studies, only the data on babies up to 18 weeks of age have been used. Altogether 275 recordings were available from 152 babies studied at ages from 3 to 18 weeks. As there was only a weak negative correlation between age at the time of study and the level of insulation used $(r-0 \cdot 16)$ and no significant correlation between age and bedroom temperature $(r-0.09)$, we have considered all the studies together rather than stratifying them by age. For the majority of the studies (174/275) the babies were put down to sleep on their sides with the remainder supine (31/275) or prone (70/275). However, the amount of insulation used did not vary significantly between positions. The recordings were evenly spread throughout the months of the year.

The bedroom temperatures recorded varied widely from 6 to $32^{\circ} \mathrm{C}$. However, within an individual study the temperature remained

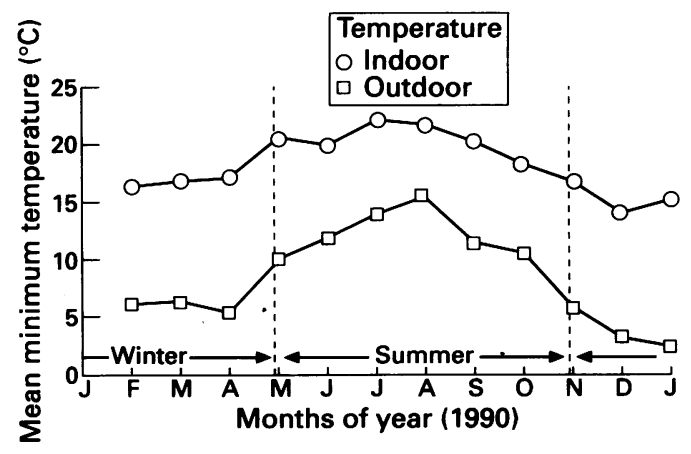

Figure 1 Monthly variation in indoor and outdoor minimum temperatures in Avon. 


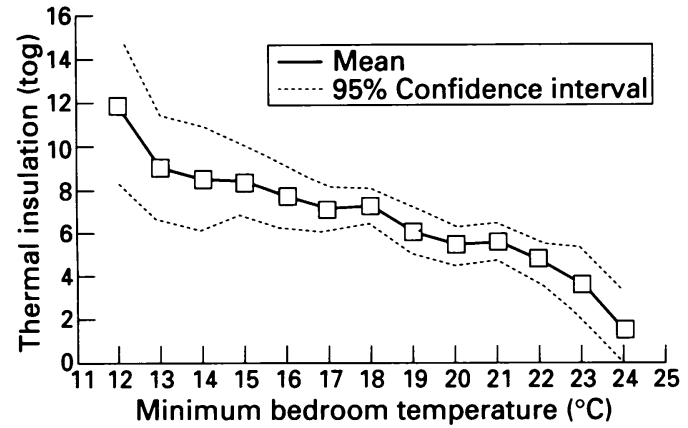

Figure 2 Relationship between the amount of added thermal insulation chosen by parents for babies at night and the minimum bedroom temperature.

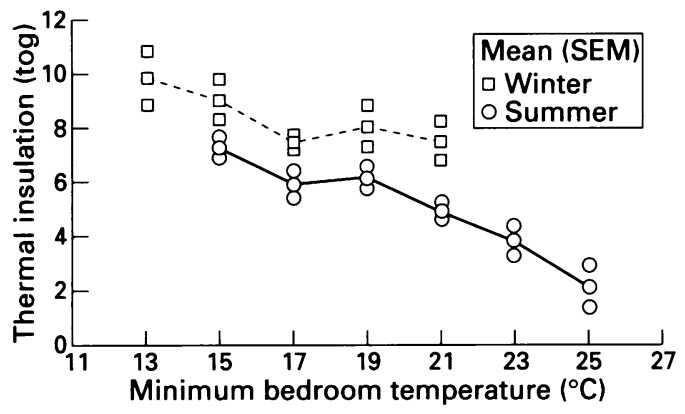

Figure 3 Seasonal differences in the amount of thermal insulation chosen for babies at night over a range of minimum overnight bedroom temperatures.

fairly constant with a median range of $2 \cdot 0^{\circ} \mathrm{C}$. Temperatures were typically highest around the baby's bedtime and lowest about eight hours later.

A marked seasonal variation was found in the indoor bedroom temperatures reflecting the pattern of outdoor temperature throughout the year (fig 1). The year divides neatly into six warmer months (May to October) and six cooler months (November through to April) and we have used this division to separate the studies into summer recordings and winter ones. The mean maximum bedroom temperature was $22.3^{\circ} \mathrm{C}$ in the

Table 1 Babies for whom satisfactory steady state recordings were obtained at environmental temperatures within the thermoneutral range $\left(23-27^{\circ} \mathrm{C}\right)$ for added insulation of 2.5 tog as assessed from standard charts

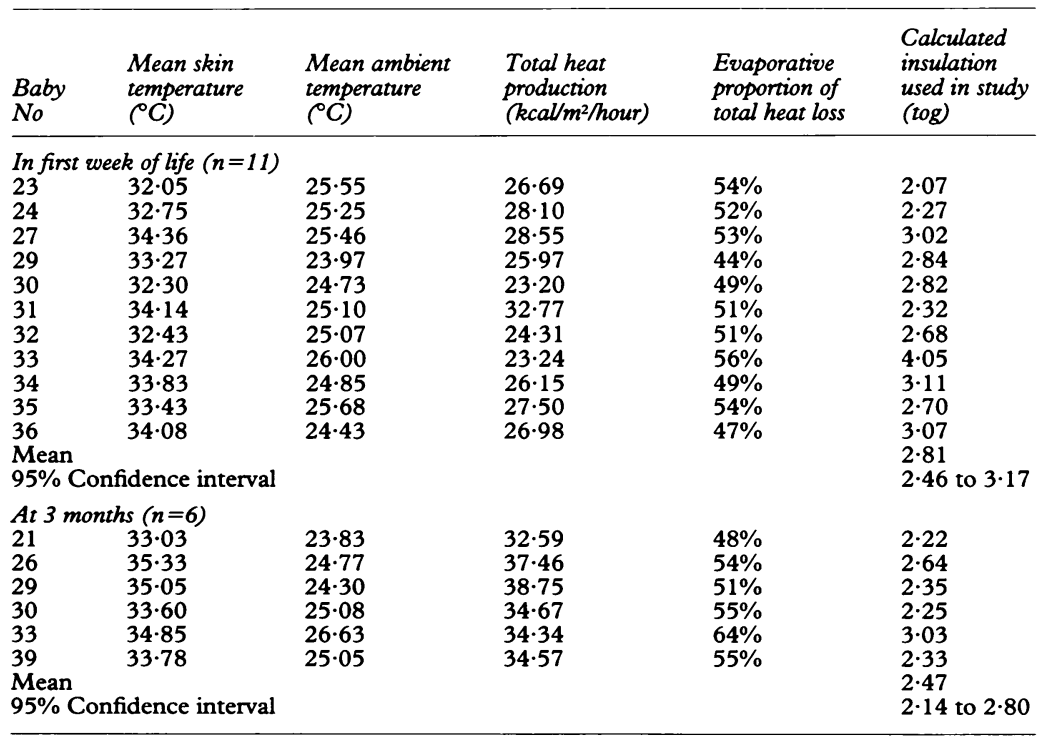

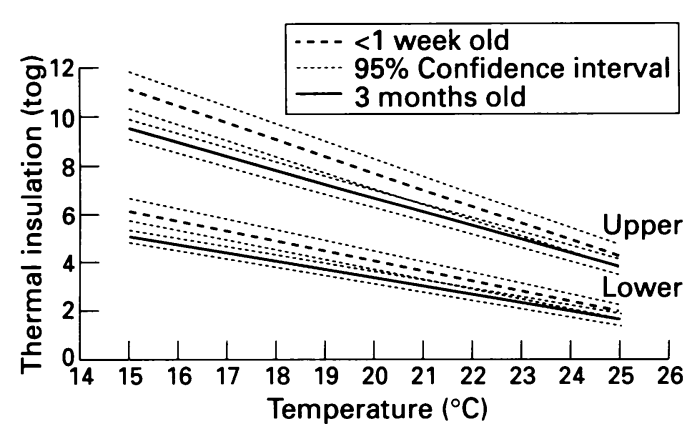

Figure 4 Calculated levels of thermal insulation required for babies up to 3 months of age to achieve thermoneutrality over a range of air temperature. Lower series: to achieve the lower end of the thermoneutral range; upper series: for a position close to the upper end of the thermoneutral range (see text).

summer and $18.8^{\circ} \mathrm{C}$ in the winter and the mean minimum temperatures were 20.2 and $16 \cdot 1^{\circ} \mathrm{C}$ respectively.

The amount of clothing and bedding used also varied widely from a baby wearing just a vest without any other covers $(0 \cdot 1$ tog) to one wearing a vest, Babygro and cardigan, who was swaddled in two doubled blankets and then placed both inside a padded Babynest and underneath an adult duvet giving a total effective insulation of about 23 tog. Very few babies slept with their heads covered.

An inverse relationship was found between the level of insulation and the minimum bedroom temperature (fig 2). A similar relationship was found with the average and the maximum bedroom temperatures. When the summer and winter values are plotted separately an interesting seasonal difference emerges (fig 3); for a particular temperature the insulation used was consistently about 2 tog greater in the winter months than in the summer. So paradoxically the babies were being kept warmer in the winter.

\section{LABORATORY RESULTS}

A summary of the values obtained in the laboratory studies are shown in table 1. The insulation values for bedding and clothing used, calculated from the heat balance equations, were close to the value of insulation known to have been used $(2 \cdot 5 \operatorname{tog})$, confirming the validity of these heat balance equations under these conditions.

At $23^{\circ} \mathrm{C}$, an environmental temperature close to the lower end of the thermoneutral range, the mean evaporative water loss within the first week of life was $17.6 \mathrm{mg} / \mathrm{kg} / \mathrm{min}$ and at 3 months of age it was $24 \cdot 1 \mathrm{mg} / \mathrm{kg} / \mathrm{min} .{ }^{4}$ The heat thus lost by evaporation at this point $(10 \cdot 1$ and $15.5 \mathrm{kcal} / \mathrm{m}^{2} /$ hour respectively) accounted for $38 \%$ of the total heat production in the first week of life and $44 \%$ at 3 months. Similarly at $27^{\circ} \mathrm{C}$, the highest temperature consistently identified as being within the thermoneutral range, ${ }^{4}$ the mean evaporative heat losses were $16.7 \mathrm{kcal} / \mathrm{m}^{2} /$ hour (first week) and 23.4 $\mathrm{kcal} / \mathrm{m}^{2} /$ hour (3 months), accounting for $63 \%$ and $66 \%$ of the total heat loss respectively.

The levels of thermal insulation calculated to achieve thermoneutrality over a range of air 


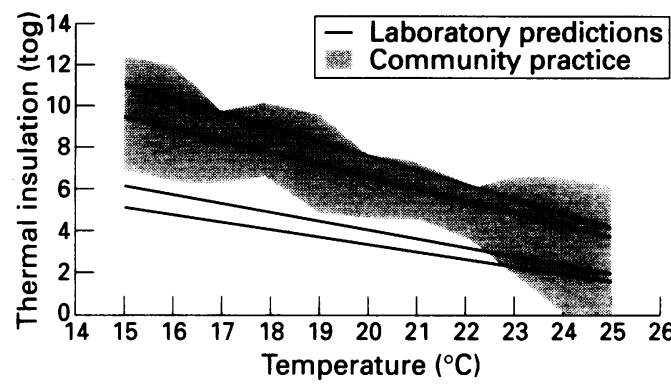

Figure 5 Comparison of the community practice with the predicted thermal insulation levels required to achieve

thermoneutrality. Community range: from the lower $95 \%$ confidence interval for the mean insulation at minimum overnight bedroom temperatures to the upper $95 \%$

confidence interval for the mean insulation at maximum overnight temperatures (see text). Laboratory predictions: (see fig 4 for 1 week and 3 month old babies; lower pair: for the lower end of the thermoneutral range, upper pair: for a

position close to the upper end of the thermoneutral range.

temperatures for each age group of babies are shown in fig 4 . The lowest pair of lines show the levels of insulation necessary for the baby to remain at the lower end of the thermoneutral range and the upper lines show the amount of insulation that would place the baby close to the top of the range but still within it. ${ }^{4}$ The required insulation is consistently higher in the first week of life than for the 3 month old babies and steadily falls with increasing environmental temperature from values at $15^{\circ} \mathrm{C}$ of $6 \cdot 1-11 \cdot 1$ tog for the younger babies and 5.1-9.5 tog for the older babies to values of $2 \cdot 0-4 \cdot 2$ tog and $1 \cdot 7-3 \cdot 8$ tog respectively at $25^{\circ} \mathrm{C}$.

\section{COMPARISON OF THE LABORATORY AND}

COMMUNITY RESULTS

In fig 5 the observed levels of thermal insulation used in the home have been superimposed on to the laboratory values calculated to be necessary to achieve thermoneutrality. The range shown for the community values is limited by the upper $95 \%$ confidence interval for the mean insulation at maximum bedroom temperature and the lower $95 \%$ confidence interval for the mean insulation at minimum bedroom temperature. Within this range there is a $95 \%$ chance of finding the mean insulation used for any temperature within the overnight range. There is quite a tight correlation between the laboratory predictions and the mean community results both in terms of absolute levels of insulation and the gradient of the range with increasing temperature.

\section{Discussion}

A close similarity has been found between the average amount of clothing and bedding that parents in Avon are choosing for their babies and values calculated from the laboratory studies to be necessary to achieve thermoneutrality over the same temperature range.

The calculated values of thermal resistance are based on certain assumptions which have been detailed in the methods section. While these assumptions may be appropriate for infants in our laboratory, they may be invalidated by some of the far more variable conditions under which infants sleep at home.

ENVIRONMENT

A draughty environment will increase both the convective and evaporative components of heat loss for a set environmental temperature and humidity. Differing levels of ambient humidity will also affect water losses.

\section{CLOTHING AND BEDDING}

The evaporative water losses also depend on the absorptive properties and the vapour diffusion resistance of the fabric covering the baby, which are determined by the nature of its component fibres and by the structure of the weave. In the laboratory studies the infants were covered by cotton blankets but the bedding used in the community varied considerably. In addition to these limitations the effective insulation provided by specific bedding will vary according to its arrangement over the baby; for example whether the covers are loosely draped over the body or tucked in tightly. ${ }^{5}$ In all the laboratory studies and in the vast majority of the home ones the babies' heads remained uncovered. A large proportion of a baby's heat loss may be through the head ${ }^{6}$ and covering it may profoundly affect the thermal balance of the baby. ${ }^{7}$ The characteristics of the mattress may also be important particularly if it is thin or not covered by an impermeable layer.

POSTURE

All the infants in our laboratory studies were lying prone. The recent evidence linking the prone sleeping position with the sudden infant death syndrome has led to a marked reduction in the proportion of babies sleeping prone in our community. ${ }^{8}$ There is some evidence that net heat loss is greater for infants sleeping supine when the area in contact with the mattress is less $(15 \%)$ than when prone $(21 \%) .{ }^{9}$ In addition, infants in a crouched or curled up prone position lose less heat than when stretched out, largely due to increased areas of skin to skin contact that reduce the surface area available for heat exchange to the environment. ${ }^{10}$

Despite all these limitations however, the calculated values obtained from our laboratory studies give some indication of the likely ranges of added insulation necessary to achieve thermoneutrality at different environmental temperatures commonly found in the home. The lower range should indicate the minimum insulation necessary, but it must be remembered that the higher predicted range does not represent the upper limit but some point below it. The maximum levels of insulation that could be tolerated without heat stress cannot be predicted from this work because the laboratory temperatures used were within the thermoneutral range and therefore the upper limit of this range was not defined.

Values for the calculated insulation required to attain 'thermal equilibrium' over a range of 
environmental temperatures have been published recently. ${ }^{3}$ They have been derived from a theoretical model of thermal balance in infants and range from $7 \cdot 2$ tog at $8^{\circ} \mathrm{C}$ to 1.5 tog at $28^{\circ} \mathrm{C}$. Between 15 and $25^{\circ} \mathrm{C}$, the environmental temperature range we have used, our predicted values for the lower end of thermoneutrality are within 0.5 tog of these theoretical values for thermal equilibrium. We have confined our predictions to this range as we are confident, from studies performed at temperatures throughout this range, that within it there is a linear relationship between the insulation required and the environmental temperature. At more extreme temperatures this relationship is likely to become non-linear due to both the degree of vasoconstriction or vasodilatation in the uncovered skin over the head and also, at the colder end, the increased temperature difference between inspired and expired gases.

From the work of Wailoo et al ${ }^{6}$ and our own studies of babies' body temperature overnight we know that most babies can tolerate a wide range and combination of environmental temperature and insulation levels without altering their core temperature patterns. They may not be in thermoneutral conditions but they are able to lose or conserve sufficient heat to remain in thermal balance. However, most of these studies were done when the babies were well. In other circumstances, such as during an acute pyrexial illness when thermoregulatory mechanisms are already disturbed with a rise in metabolic rate and peripheral vasoconstriction, ${ }^{11}$ the range of environmental thermal conditions that a baby can cope with may be much narrower with an increased vulnerability to heat stress. The combination of both heavy wrapping and an acute viral infection has been shown to carry a substantially higher risk of sudden death than either factor alone. ${ }^{12}$

Although the maintenance of thermoneutrality has been shown to improve the outcome for sick infants, ${ }^{13}$ there is no evidence of benefit from such thermal conditions in normal healthy infants. One might expect that the lower oxygen consumption of the thermoneutral range would allow more energy to be channelled into body growth with improved weight gain but there is no evidence that this is true for healthy infants. As the evaporative water losses increased significantly across the whole of the thermoneutral range, there might in fact be some benefit in

Table 2 Thermal guidelines for babies up to 4 months

\begin{tabular}{|c|c|c|c|c|c|}
\hline $\begin{array}{l}\text { Season } \\
\text { of night }\end{array}$ & $\begin{array}{l}\text { Usual range } \\
\text { of overnight } \\
\text { room temperature* }\end{array}$ & Tog & $\begin{array}{l}\text { Clothing } \\
\text { ( } 0.8 \text { tog) }\end{array}$ & $\begin{array}{l}\text { Sheet } \\
\text { (0.2 tog) }\end{array}$ & $\begin{array}{l}\text { Layers of } \\
\text { blanket } \\
(1 \cdot 2 \text { tog/ayer })\end{array}$ \\
\hline $\begin{array}{l}\text { Winter (cool) } \\
\text { Winter (average) } \\
\text { Summer (average) } \\
\text { Summer (warm) }\end{array}$ & $\begin{array}{l}14-17^{\circ} \mathrm{C} \\
16-19^{\circ} \mathrm{C} \\
20-22^{\circ} \mathrm{C} \\
23-25^{\circ} \mathrm{C}\end{array}$ & $\begin{array}{l}6-9 \\
5-8 \\
4-6 \\
3-4\end{array}$ & $\begin{array}{l}\text { Babygro, vest } \\
\text { Babygro, vest } \\
\text { Babygro, vest } \\
\text { Babygro, vest }\end{array}$ & $\begin{array}{l}1 \\
1 \\
1 \\
1\end{array}$ & $\begin{array}{l}4-7 \\
4-6 \\
3-4 \\
2-3\end{array}$ \\
\hline \multicolumn{6}{|c|}{$\begin{array}{l}\text { Leave head uncovered } \\
\text { Check baby regularly and adjust bedding if baby is hot or cold } \\
\text { Reduce insulation if baby has a fever }\end{array}$} \\
\hline
\end{tabular}

For more extreme temperatures such as colder winter nights or hotter summer ones, insulation should be respectively increased or decreased beyond the values given here, with careful subsequent adjustment for the individual baby's response.

^ Based on temperatures in Avon. remaining closer to the lower end of the range than higher up within it. However, the question of whether the thermoneutral temperature range represents the optimal temperature range for healthy infants still remains uncertain.

The results of the community studies may help to identify the factors considered by parents when deciding what thermal conditions are appropriate for their babies. The similarity between the laboratory estimates and the observed levels of insulation used in the home suggests that the 'formula' parents are using aims to achieve thermoneutral conditions. Although there was a clear relationship found between the bedroom temperature and applied insulation there was a wide scatter of tog values for each temperature suggesting that additional factors are involved in the choice of insulation. The seasonal differences in insulation levels for the same minimum temperatures, with more bedding applied in the winter months, may mean that the parents' perception of expected weather conditions and likely outside temperatures also plays a part in their decision making. Social circumstances may also contribute as there is some evidence that babies in socially disadvantaged households are likely to be kept warmer than those in more affluent ones. ${ }^{214}$ Moreover, although $20 \%$ of mothers in both Newcastle ${ }^{14}$ and Exeter ${ }^{15}$ felt it was appropriate to keep their baby warmer than usual during a febrile illness, this belief was most common in the younger and less educated mothers.

As we have shown, on average, the parents in our study seem to instinctively provide appropriate thermal conditions for their babies to sleep in. We do not want infant care to become 'so scientific' that parents ignore those instincts and rely on temperature and tog charts, but broad, scientifically based guidelines (table 2) would be useful for parents and health professionals to refer to when unsure or in need of reassurance. The baby's head should be left uncovered and the amount of chosen bedding should be individually adjusted according to the particular baby's reactions; removing a layer if the baby feels hot or sweaty and adding additional covers if cool. We would also emphasise the importance of explaining to parents that when their baby has a fever, wrappings should not be increased and may need to be reduced.

\section{SUMMARY}

On average, parents in Avon are providing appropriate conditions for their babies to sleep in thermal comfort. We propose broad thermal guidelines for the young baby based on both common practice within our community and laboratory values calculated to achieve thermoneutrality.

We are grateful to the Foundation for the Study of Infant Deaths for their support of the community study and to both Action Research for the Crippled Child and Cot Death Mesearch for supporting the laboratory work. We also thank Mike Levine for his help in the laboratory studies, Professor and the parents and babies of Avon for their cooperation. 


\section{Appendix}

Under conditions of thermal balance the total heat lost should equal the total heat produced (metabolic rate). The effective thermal insulation of the clothing and the bedding for the baby equals the temperature difference between the skin and the air divided by the dry heat loss per unit surface area:

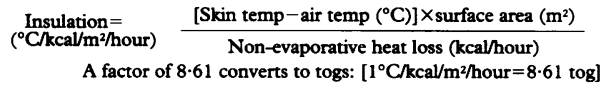

The surface area of each baby was calculated using the formula of Haycock et al. ${ }^{16}$ The heat lost by conduction through a thick mattress has been shown to be very small ${ }^{10}$ and thus the skin in contact with the mattress is considered unavailable for heat exchange. This area varies with position and Stothers and Warner have shown it to be $21 \%$ for infants lying prone. ${ }^{10}$ Thus in our studies $79 \%$ of the baby's surface area was theoretically available for heat exchange.

The metabolic rate was calculated from the continuous measurements of oxygen consumption. The evaporative heat loss was calculated from the measured total evaporative water loss assuming a latent heat of vaporisation of $580 \mathrm{kcal} / 1$ between 31 and $35^{\circ} \mathrm{C}^{17}$ and the relative proportions of the total heat loss due to evaporation and dry heat loss were determined for each study temperature.

In addition to the clothing and bedding, the layer of still air surrounding the baby also contributes to the effective thermal insulation. Under draught free conditions the insulation of this air layer has been shown to be approximately 1.5 tog. ${ }^{18} 19$ Taking this into account, the heat balance equation now becomes:

$$
\begin{aligned}
& \text { Insulation of } \\
& \text { bedding and } \\
& \text { clothing (tog) }
\end{aligned}=\frac{\left[\text { Skin temp-air temp }\left({ }^{\circ} \mathrm{C}\right)\right] \times 8.61}{\text { Non-evaporative heat loss }\left(\mathrm{kcal} / \mathrm{m}^{2} / \mathrm{hour}\right)}-1.5
$$

Using the measured values of skin temperature, air temperature, non-evaporative heat loss and surface area, the above equation was solved for each infant to determine the tog value of the bedding and clothing actually used in the study (approximately 2.5 tog excluding the nappy). A series of air temperatures between $15-25^{\circ} \mathrm{C}$ were then substituted into the equation to determine the amount of insulation needed at each temperature to allow the baby to remain within the thermoneutral range. The non-evaporative heat loss at the identified lower end of the range was used to find the minimum insulation for thermoneutrality and its value at a higher temperature still within the range was used to determine higher levels of insulation under which thermoneutrality could still be achieved.

1 Stanton AN. Overheating and cot death. Lancet 1984; ii: 1199-201.

2 Fleming PJ, Gilbert $R$, Azaz $Y$, et al. The interaction between bedding and sleeping position in the sudden infant syndrome: a population based controlled study. BMF 1990; 301: 85-9

3 Ponsonby A-L, Dwyer T, Gibbons LE, Cochrane JA, Jones ME, McCall MJ. Thermal environment and sudden infant death syndrome: case-control study. $B M \mathcal{F} 1992$; 304: 277-82.

4 Azaz Y, Fleming PJ, Levine M, McCabe R, Stewart A, Johnson $P$. The relationship between environmental temperature, metabolic rate, sleep state and evaporative water loss in infants from birth to three months. Pediatr Res 1992; 32: 417-23.

5 Kerslake DMcK. The insulation provided by infants' bedclothes. Ergonomics 1991; 34: 893-907.

6 Wailoo MP, Petersen SA, Whittaker H, Goodenough P. Sleeping body temperatures in three-four month old infants. Arch Dis Child 1989; 64: 596-9.

7 Stothers JK. Head insulation and heat loss in the newborn. Arch Dis Child 1981; 56: 530-4.

8 Wigfield RE, Fleming PJ, Berry PJ, Rudd PT, Golding J. Can the fall in Avon's sudden infant death rate be explained by changes in sleeping position? BMF 1992; 304: 282-3.

9 Nelson EAS, Taylor BJ, Weatherall IL. Sleeping position and infant bedding may predispose to hyperthermia and the sudden infant death syndrome. Lancet 1989; i: 199-201.

10 Stothers JK, Warner RM. Thermal balance and sleep state in the newborn. Early Hum Dev 1984; 9: 313-22.

11 Fleming PJ, Levine MR, Azaz Y, Wigfield RE, Stewart AJ. Interactions between thermoregulation and the control of respiration in infants: possible relationship to sudden respiration in infants: possible rela

12 Gilbert R, Rudd P, Berry PJ, et al. Combined effect of infection and heavy wrapping on the risk of sudden unexpected infant death. Arch Dis Child 1992; 67: 171-7.

13 Silverman WA, Fertig JW, Berger AP. The influence of the thermal environment upon the survival of newly born premature infants. Pediatrics 1958; 22: 876-86.

14 Bacon CI, Bell SA, Clulow EE, Beattie AB. How mothers keep their babies warm. Arch Dis Child 1991; 66: k27-32.

15 Eiser C, Town C, Tripp J. Dress and care of infants in health and illness. Arch Dis Child 1985; 60: 465-70.

16 Haycock GB, Schwartz GJ, Wisotsky DH. Geometric method for measuring body surface area. A height-weight formula validated for infants, children and adults. F Pediatr 1978; 93: 62-6.

17 Kaye GWC, Laby TH. Tables of physical and chemical constants. 15th Ed. New York: Longman, 1986: 268.

18 Winslow CEA, Gagge AP, Herrington LP. The influence of air movement upon heat losses from the clothed human air movement upon heat losses from the

19 Renbourn ET, Rees WH. Materials and clothing in health and disease. London: H K Lewis, 1972: 131-7. 